\title{
A Unique Model for ONSD Part II: Inter/Intra-operator Variability
}

\author{
F.A. Zeiler, B. Unger, Q. Zhu, J. Xiao, A.W. Kirkpatrick, A.H. Kramer, \\ E.M. Aleassa, L.M. Gillman
}

\begin{abstract}
Objective: To evauluate our novel ultrasound model for measurement of optic nerve sheath diameter (ONSD) and determine the intra- and inter-operator variability associated with this technique. Methods: We conducted ten measurements of ONSD per model amongst eight different models with a single experienced operator to examine intra-operator variability. Similarly, we had seven different operators measure the OSND twice in eight different models, in order to determine inter-operator variability analyzed with a three level linear statistical model. Results: For intra-operator variability, the intra-cluster correlation coefficients for the experienced and novice operators were 0.643 and 0.453 respectively. This displayed improvement in intra-operator variability with experience. The inter-cluster correlation coefficient was 0 for the group of novice operators, indicating negligible difference amongst multiple operators in measuring any given model of ONSD. A strong, statistically significant, linear relationship between the actual model disc size and the ultrasound ONSD measures was identified, implying the reliability of the images produced by our novel model. Conclusions: Utilizing a novel model for ONSD ultrasonography, we have determined the intraoperator reliability of ONSD measurement to be moderate, with no appreciable difference amongst multiple operators. Improvement in measurement reliability has been demonstrated between expert and novice operators with our model, indicating the potential benefit of simulation platforms for teaching the technique of ONSD ultrasound.
\end{abstract}

RÉSUMÉ: Un modèle unique de mesure du diamètre de la gaine du nerf optique, 2e partie : variabilité inter/intra opérateur. Objectif : Le but de cette étude était d'évaluer notre nouveau modèle de mesure du diamètre de la gaine du nerf optique (DGNO) par ultrasons et de déterminer la variabilité intra et inter opérateur associée à cette technique. Méthode : Un même opérateur d'expérience a effectué dix mesures du DGNO par modèle sur huit modèles différents afin de déterminer la variabilité intra opérateur. Nous avons également demandé à sept opérateurs différents de mesurer le DGNO à deux reprises sur huit modèles différents afin d'évaluer la variabilité interopérateur. Un modèle statistique linéaire à trois niveaux a été utilisé pour analyser les données. Résultats : Pour la variabilité intra-opérateur, les coefficients de corrélation intragrappes chez les opérateurs d'expérience et les novices étaient de 0,643 et 0,453 respectivement, ce qui démontrait que la variabilité intra-opérateur s'était améliorée avec l'expérience. Le coefficient de corrélation intergrappe était de zéro chez le groupe d'opérateurs novices, indiquant une différence négligeable entre les multiples opérateurs, quel que soit le modèle de DGNO. Nous avons observé une relation linéaire très significative au point de vue statistique entre la taille réelle du disque et les mesures à l'ultrasonographie, ce qui témoigne de la fiabilité des images produites par notre nouveau modèle. Conclusions : Au moyen d'un nouveau modèle d'ultrasonographie du DGNO, nous avons déterminé que la fiabilité intra-opérateur de la mesure du DGNO était modérée, sans avoir constaté de différence appréciable parmi les multiples opérateurs. Une amélioration de la fiabilité des mesures a été démontrée entre les opérateurs experts et novices, indiquant que l'utilisation de plateformes de simulation pour l'enseignement de la technique d'ultrasonographie du DGNO pourrait comporter un bénéfice.

Can J Neurol Sci. 2014; 41: 430-435

Optic nerve sheath diameter ultrasonography as a means for noninvasive determination of intracranial pressure (ICP) is a technique recently receiving consideration in the intensive care and emergency medicine literature ${ }^{1-4}$. The concept of a noninvasive, readily available technique to perform at-thebedside, point-of-care determination of ICP is an attractive means of early identification and potentially objective management of intracranial hypertension, especially in resourceconstrained or austere environments.

Current usage of ultrasound for optic nerve sheath diameter (ONSD) has focused on perioperative assessment in the neurosurgical community, as well as both intensive care unit (ICU) and emergency department (ED) based correlations to invasive dynamical intracranial ICP monitoring. Pre- and postoperative assessment of ONSD in neurosurgical patients, particularly in those patients with shunt dependent hydrocephalus, has yielded data suggesting an increased ONSD in those with shunt malfunction which subsequently responds to shunt revision ${ }^{5}$. Similarly, ICU and ED literature has confirmed

From the Section of Neurosurgery (FAZ), Section of General Surgery (EMA, LMG), Department of Surgery, Department of Medical Education (BU), University of Manitoba, Winnipeg, Manitoba; Section of Neurocritical Care (FAZ), Montreal Neurological Institute, Mcgill University, Montreal, Quebec; Regional Trauma Services and Section of General Surgery, Department of Surgery (QZ, JX, AWK), Section of Critical Care Medicine (AWK, AHK), Department of Clinical Neurosciences (AHK), University of Calgary, Calgary, Alberta, Canada. Received November 21, 2013. Final Revisions Submitted January 8, 2014 Correspondence to: Frederick A. Zeiler, Section of Neurosurgery, Dept of Surgery, University of Manitoba, GB-1 Health Sciences Center, 820 Sherbrook Street, Winnipeg, Manitoba, R3A 1R9, Canada.Email: umzeiler@cc.umanitoba.ca. 
responsiveness of ONSD to fluctuations in ICP, suggesting the validity of the concept and to some supporting further use of this technique $^{6,7}$.

Despite these promising results with the technique of ONSD ultrasonography in vivo, concerns surrounding the normal ranges for ONSD, accuracy of measurements, variation between observers, and reliability of changes in ONSD in response to changes in ICP have been raised.

Given the aforementioned concerns about the current literature supporting the use of ONSD ultrasound for determination of ICP, we perceived a need to construct a novel model for research and teaching ONSD ultrasound ${ }^{8}$. The necessary criteria were to be able to display realistic ultrasound images that closely resemble those in vivo ${ }^{8}$. Our goal was thus to create a model to determine the intra- and inter-operator variability of ultrasound for ONSD in a controlled environment, in order to properly define the difficulties with this technique and guide further ultrasound training by constructing a platform for practice and instruction.

Within this manuscript, we describe the second phase of our study where we first evaluate the model with ultrasound and then utilize it to define intra- and inter-operator variability with ultrasound for ONSD.

\section{METHODS}

\section{Model Construction}

Utilizing our previously described techniques, we created models of the globe and optic nerve sheath complex out of gelatin and 3D printed (Zprinter 650, 3D Systems, Rock Hill, $\mathrm{SC})$ resin-coated plaster discs ${ }^{8}$. The globes were formed using a one inch spherical baking mold (Chicago School of Mold Making, Oak Park, Il). The 3D printer discs were made to $2 \mathrm{~mm}$ thickness with varying diameters, and the acoustic shadow produced by these represent the entire optic nerve complex in the model. Various colored dyes were added to the 3D discs in order to distinguish between disc sizes. The color-coding was blinded to all operators throughout both phases of the study. All models were placed in a background of gelatin which had sugar-free psyllium powder added to provide acoustic contrast between model and background. An example of our model and the ultrasound technique can be seen in the Figure.

For measuring intra-operator variability with an expert operator, we constructed eight different models with disc sizes measuring: 2.30 (A), 4.90 (B), 5.12 (C), 5.22 (D), 5.34 (E), 5.44 (F), $5.54(\mathrm{G})$ and $7.70(\mathrm{H}) \mathrm{mm}$. All models were assigned letters. These eight different discs sizes were specifically chosen to represent the lower to upper limit of normal ONSD measurements previously defined in the literature ${ }^{3}$ with the majority of discs (five) clustered around 5 to $5.5 \mathrm{~mm}$, as this has been described as the cutoff for raised $\mathrm{ICP}^{2}$. In doing so we hoped to determine the operators ability to discern amongst models of similar ONSD.

For determining inter-operator variability, we again constructed eight models with ONSD measuring: 2.30 (I), 2.90 (II), 3.50 (III), 4.00 (IV), 4.50 (V), 6.00 (VI), 7.00 (VII), and 7.70 (VIII) mm. All models were assigned Roman numerals.

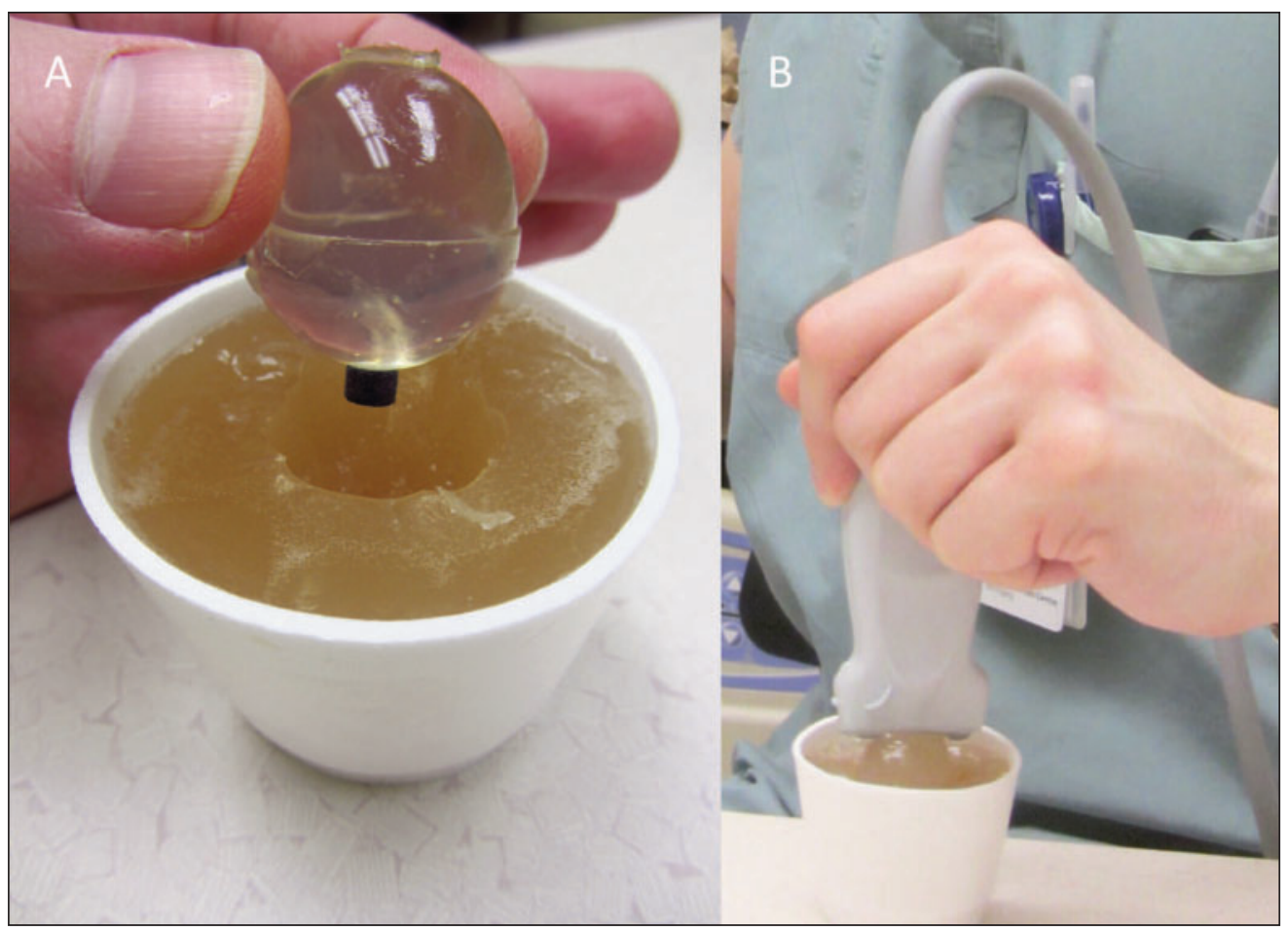

Figure: Model of Optic Nerve Sheath Diameter. A) Gelatin globe with plaster disc attached simulating the optic nerve sheath. The psyllium augmented gelatin background can be seen in the Styrofoam cup. B) Standard linear array vascular ultrasound probe is held perpendicular to model construct for image acquisition. 
These disc sizes were also chosen to represent the range of the previously defined normal spectrum.

\section{Ultrasound Technique}

Using standard ultrasound techniques for measuring ONSD $^{1,9-13}$, we utilized the 13-6 MHz linear array ultrasound transducer (L25x transducer, Sonosite Corp, Bothell, WA) and a portable ultrasound unit (Sonosite M-Turbo, SonoSite Inc, Bothell, WA) to conduct all ONSD measurements throughout the study. The ultrasound probe was held perpendicular to the model construct and using a small amount of ultrasound gel, images were acquired. Once the globe and shadow created by the $3 \mathrm{D}$ disc (representing the optic nerve sheath) were displayed, the diameter of the disc shadow was measured at $3 \mathrm{~mm}$ behind the globe ${ }^{8}$. This diameter was recorded as the ONSD for the model.

\section{Intra-operator Variability}

Our initial trials suggested an intra-cluster correlation coefficient (ICC) of 0.24 for intra-rater reliability of a single rater. Based on these initial trials and assuming the true value of ICC may be as high as 0.9 , we calculated we would require eight different discs with ten measurements per disc by a single blinded rater to achieve a power of $80 \%$ when calculating the true intra-rater ICC. A single operator, experienced in the measurement of ONSD, both in vivo and with our model, was used for the entirety of this part of the study. We aimed to determine intra-operator variability and any linear relationship between disc shadow measurement and actual disc size.

\section{Inter-operator Variability}

Utilizing the assumption that the inter-rater reliability would be similar to the intra-rater reliability, we calculated we would require seven raters measuring eight different discs with two measurements per disc to achieve a power of $80 \%$ to calculate the true inter-rater ICC. Using the previously mentioned eight models for ONSD constructed for assessment on inter-operator variability, two measurements were made per model. Models with disc sizes within a mm of one another were selected in order to represent sizes of literature described "normal", and to evaluate the technique with more difficult to discern representations of ONSD. A total of seven different operators were used. All of these operators, except one, were considered novice with the technique of ONSD ultrasound, yet all had experience with point-of-care critical care ultrasound.

\section{Statistical Analysis}

All the statistical analysis was performed using SAS 9.2 (SAS Institute, Cary, NC), using the Proc Mixed procedure. For the intra-operator variability analysis, we used a two-level linear model with measurements nested under disc, and the sizes of the disc as the only independent variable. The intra-operator correlation coefficient (ICC) was calculated as the percentage of variation due to the disc size over total variation. A linear regression model was used to assess correlation between disc acoustic shadow and actual disc size. For the analysis of interoperator variability, we utilized a three-level hierarchical linear model, with Operator at the top level, disc size at the middle level and the measurement at the lowest level, and the disc size as the only independent variable. The inter-operator correlation coefficient was calculated as the percentage of variation due to operator (top level) over total variation, and the intra-operator correlation coefficient is the percentage of variation due to disc size (level 2) over total variation.

Correlation at the level of the measurement represents the relationship of the acoustic shadow measure to the object of interest, the disc diameter. Correlation at the level of the disc represents the reliability of repeat measurement of the same model in any given operator. Finally, correlation at the level of the operator refers to the relationship between multiple operators on a given model, and described correlations of measurements between operators.

\section{Ethics}

This study received approval from the University of Manitoba Health Research Ethics Board.

Table 1: Intra-operator variability disc diameters and mean measurements

\begin{tabular}{|c|c|c|c|c|}
\hline Model & $\begin{array}{c}\text { Actual Disc } \\
\text { Diameter }(\mathrm{mm})\end{array}$ & $\begin{array}{c}\text { Mean ONSD } \\
\text { Measurement } \\
(\mathrm{mm}) \\
\end{array}$ & $\begin{array}{c}\text { Standard Deviation } \\
\text { of Measurements } \\
(+/-\mathrm{mm})\end{array}$ & $\begin{array}{c}\text { Difference Between Actual } \\
\text { and Measured (mm) }\end{array}$ \\
\hline A & 2.30 & 1.81 & 0.10 & -0.50 \\
\hline B & 4.90 & 4.46 & 0.28 & -0.44 \\
\hline C & 5.12 & 4.80 & 0.14 & -0.32 \\
\hline D & 5.22 & 4.88 & 0.15 & -0.34 \\
\hline $\mathrm{E}$ & 5.34 & 4.89 & 0.17 & 0.45 \\
\hline $\mathbf{F}$ & 5.44 & 4.72 & 0.18 & -0.72 \\
\hline G & 5.54 & 5.01 & 0.16 & -0.53 \\
\hline $\mathbf{H}$ & 7.70 & 7.90 & 0.08 & +0.20 \\
\hline
\end{tabular}

$\mathrm{mm}=$ millimeter, $\mathrm{ONSD}=$ optic nerve sheath diameter 
Table 2: Inter-operator disc measurements

\begin{tabular}{|c|c|c|c|c|c|c|c|c|}
\hline & $\begin{array}{c}\text { Large Black } \\
(0.77 \mathrm{~cm})\end{array}$ & $\begin{array}{c}\text { Large Red } \\
(0.49 \mathrm{~cm}) \\
\end{array}$ & $\begin{array}{c}\text { Small Black } \\
(0.23 \mathrm{~cm})\end{array}$ & $\begin{array}{l}\text { Small Red } \\
(0.29 \mathrm{~cm}) \\
\end{array}$ & $\begin{array}{c}\text { Blue }(0.70 \\
\mathbf{c m})\end{array}$ & $\begin{array}{c}\text { Dark Green } \\
(0.40 \mathrm{~cm})\end{array}$ & $\begin{array}{c}\text { Lime Green } \\
(0.35 \mathrm{~cm})\end{array}$ & $\begin{array}{c}\text { Purple } \\
(0.60 \mathrm{~cm})\end{array}$ \\
\hline \multirow[t]{2}{*}{ Operator A } & 0.72 & 0.38 & 0.22 & 0.27 & 0.66 & 0.35 & 0.29 & 0.53 \\
\hline & 0.74 & 0.41 & 0.23 & 0.29 & 0.68 & 0.38 & 0.33 & 0.54 \\
\hline \multirow[t]{2}{*}{ Operator B } & 0.72 & 0.56 & 0.28 & 0.24 & 0.59 & 0.36 & 0.52 & 0.54 \\
\hline & 0.77 & 0.54 & 0.25 & 0.27 & 0.64 & 0.34 & 0.4 & 0.59 \\
\hline \multirow[t]{2}{*}{ Operator C } & 0.76 & 0.46 & 0.22 & 0.28 & 0.62 & 0.32 & 0.36 & 0.52 \\
\hline & 0.74 & 0.42 & 0.24 & 0.24 & 0.66 & 0.32 & 0.38 & 0.58 \\
\hline \multirow[t]{2}{*}{ Operator D } & 0.76 & 0.4 & 0.18 & 0.28 & 0.71 & 0.39 & 0.28 & 0.6 \\
\hline & 0.96 & 0.44 & 0.23 & 0.22 & 0.67 & 0.29 & 0.38 & 0.54 \\
\hline \multirow[t]{2}{*}{ Operator E } & 0.82 & 0.38 & 0.2 & 0.28 & 0.7 & 0.32 & 0.36 & 0.56 \\
\hline & 0.76 & 0.4 & 0.22 & 0.22 & 0.66 & 0.34 & 0.34 & 0.56 \\
\hline \multirow[t]{2}{*}{ Operator F } & 0.76 & 0.53 & 0.2 & 0.26 & 0.58 & 0.35 & 0.31 & 0.54 \\
\hline & 0.79 & 0.5 & 0.21 & 0.24 & 0.66 & 0.32 & 0.32 & 0.52 \\
\hline \multirow[t]{2}{*}{ Operator G } & 0.75 & 0.42 & 0.22 & 0.24 & 0.65 & 0.34 & 0.32 & 0.51 \\
\hline & 0.73 & 0.44 & 0.21 & 0.27 & 0.68 & 0.33 & 0.34 & 0.55 \\
\hline
\end{tabular}

Letters A-G represent operator designation. Size in brackets beside disc name represents actual disc measure. $\mathrm{cm}=$ centimeters.

\section{RESULTS}

\section{Intra-operator Variability}

The mean for the ten measurements of ONSD are shown in Table 1. The intra-cluster correlation coefficient value for intraoperator variability was determined to be 0.643 .

Throughout the analysis of the mean measured ONSD and the actual disc size, we were able to determine a linear relationship between the measured shadow size on ultrasound and the actual $3 \mathrm{D}$ disc diameter. This relationship could be expressed as:

$$
\text { Measured Shadow Size }(\mathrm{mm})=(1.11 \text { x actual disc size })-0.1
$$

\section{Inter-operator Variability}

For the inter-operator variability portion of the study, seven operators were used to measure eight different models of ONSD two times each (Table 2).
The mean ONSD measurements (and standard deviations) for each model I - VIII across all operators can be seen in Table 3. Overall, the accuracy of measurements can be estimated as +/0.11 to $0.22 \mathrm{~mm}$ across all operators.

We analyzed the data utilizing a 3-level linear statistical model, with levels at the shadow measurement, actual disc size, and the operator.

A very strong relationship was found between the actual disc diameter and the shadow as measured by ultrasound, with a regression coefficient of 0.985 ( $\mathrm{p}$-value $<0.01$ ). This indicates the shadow measured via ultrasound with our model accurately predicts the actual disc size.

At the level of the disc, with two measures occurring per model (per operator), there was moderate correlation found between measurements with an intra-cluster correlation coefficient of 0.453 . This indicates a moderate correlation between the measures of the same model of ONSD by an

Table 3: Inter-operator overall mean ONSD measurements

\begin{tabular}{cccc}
\hline Model & $\begin{array}{c}\text { Actual Disc Diameter } \\
(\mathbf{m m})\end{array}$ & $\begin{array}{c}\text { Mean ONSD } \\
\text { Measurement }(\mathbf{m m})\end{array}$ & $\begin{array}{c}\text { Standard Deviation in ONSD } \\
\text { Measurements (mm) }\end{array}$ \\
\hline \hline I & 2.30 & 2.22 & 0.24 \\
II & 2.90 & 2.57 & 0.23 \\
III & 3.50 & 3.52 & 0.59 \\
IV & 4.00 & 3.39 & 0.26 \\
V & 4.50 & 4.49 & 0.60 \\
VI & 6.00 & 5.49 & 0.26 \\
VII & 7.00 & 6.54 & 0.37 \\
VIII & 7.70 & 7.70 & 0.61 \\
\hline
\end{tabular}

$\mathrm{mm}=$ millimeter, $\mathrm{ONSD}=$ optic nerve sheath diameter 
individual operator. The remaining variation in measurements, 0.547 , is due to random measurement error.

Finally, at the top level, the level of the operator, the intercluster correlation coefficient value was 0 . Thus, multiple measures of a model by a single operator are essentially the same as one measurement taken by multiple operators of the same model.

\section{DISCUSSION}

Point-of-care ultrasound techniques have become critical in the management of ED and ICU patients. The ability to quickly obtain qualitative and quantitative data at the bedside, with ever improving image resolution has afforded us the ability to implement, with increasing confidence, aggressive management in critically ill patients prior to results of laboratory and invasive investigations.

Early objective quantification of ICP however has been difficult without invasive intracranial monitoring or cranial imaging. Given that the eyes provide "a window to the brain", the application of ultrasound for ONSD measurements and its correlation to ICP have recently been appearing in the literature. Concerns over the range of normal, cutoff values for increased ICP, intra- and inter-operator variability, reactance of the optic nerve sheath to increase in ICP, and learning curve with the technique have been raised.

To date, studies utilizing a small number of healthy control subjects have attempted to define the normal mean of $5.4 \mathrm{~mm}$ (range of $4.3-7.6 \mathrm{~mm})^{3}$. Literature on small cohorts of patients with intracranial pathology and invasive monitoring has suggested cutoff values of ONSD that predict elevated ICP to be around $5 \mathrm{~mm}$ or higher ${ }^{2}$, contradicting assessments of normal ranges for ONSD in healthy subjects ${ }^{3}$. Recent meta-analysis suggests the sensitivity and specificity of ONSD ultrasonography to detect elevated ICP at $90 \%$ and $85 \%$ respectively, suggesting up to $10 \%$ of patients with significant ICP issues will be missed with this technique ${ }^{1}$. Intra- and Interoperator variability assessments thus far have focused on small numbers of expert ultrasound operators measuring ONSD in a small number of patients. Intra-observer reliability between 0.92 and $0.97^{3}$, while inter-observer reliability with a Pearson correlation of $0.81^{3}$ and a mean inter-observer ONSD variation of 0.1 to $0.4 \mathrm{~mm}^{2-4}$ have been reported.

Given the concerns around existing literature, we attempted to assess the technique of ONSD ultrasonography utilizing the creation of a novel model and determining the intra- and interoperator variability. Our model relies on a solid disc creating an acoustic shadow that in turn recreates an image that mimics the ultrasound appearance of an optic nerve sheath ${ }^{8}$.

Previous evaluations of the model support that the resultant ultrasound images are comparable to those obtained in vivo ${ }^{8}$. Therefore in the current paper we attempted to further evaluate the models capabilities by defining the intra- and inter-operator variability with ultrasound for ONSD in our model. Our goal was to define the variability with the technique in a controlled environment in order to properly understand the difficulties with the technique and potentially provide a platform for instruction in the future.

In our analysis, there was a direct linear relationship between the actual disc size and measured ONSD shadow on ultrasound indicating the reliability of the images produced with our model via ultrasound. This was confirmed in the inter-operator variability portion of the study with a statistically significant regression coefficient of 0.985 (p-value $<0.01$ ) for disc size to measured ONSD shadow size. Both of these results implicate the potential usefulness of our model for reliable and reproducible results in a controlled setting. As a result, our model represents the first simulation platform in the literature for ONSD ultrasonography, now with validated reliability.

Three important conclusions on the technique of ONSD ultrasonography can be drawn from our data. First, we displayed an intra-cluster correlation coefficient of 0.643 for a single experienced ultrasound operator. This indicates moderate intraoperator reliability of multiple measurements with the technique of ONSD ultrasound, and demonstrates a $36 \%$ rate of random error associated with measurements. This value for the intracluster coefficient is considerably lower than that previously described in the literature by "expert" operators conducting a small number of measurements in vivo ${ }^{2}$. We believe our intracluster coefficient is lower due to the large number of measurements, and the similar disc sizes clustered around 5 to $5.5 \mathrm{~mm}$. Given the large sample size and controlled environment (excluding patient factors as seen in vivo) in our study, there is a higher likelihood that our coefficient is closer to the real value. It is quite possible, given the previous studies, that with the small sample sizes measured, the intra-cluster coefficients approaching 0.972 could be due to chance. The large number of discs with similar sizes utilized in our study was designed to simulate the "cutoff" value for elevated ICP. Given the intra-cluster correlation coefficient of 0.643 , it is possible this value stems from the difficulty with discerning among discs of similar size (less than $0.5 \mathrm{~mm}$ difference). Similarly, for novice operators the intra-cluster correlation coefficient was 0.453 across all operators, indicating a 55\% random error associated with measurements. With these results for intra-cluster correlation with a single experienced and multiple novice operators, we are able to show that there is a learning curve for ONSD ultrasonography and potential for improvement in intra-operator variability between novice and expert operators, further indicating the need for a simulation platform to guide instruction on the challenging technique. In addition, we also demonstrated the random measurement error associated with the technique to still remain significant even in the setting of an experienced operator and a controlled environment. Therefore, it can be expected that in vivo such random measurement error would likely be higher.

Second, the overall accuracy of measurements amongst all operators was $+/-0.11$ to $0.22 \mathrm{~mm}$, indicating a relatively small standard deviation between multiple measurements of ONSD in any given model. Given the currently defined ${ }^{3}$ normal range of ONSD measurements in vivo to be $4 \mathrm{~mm}$ to $7 \mathrm{~mm}$, we need high accuracy to differentiate normal from abnormal ONSD measurements. The accuracy described in our study closely resembles that previously described at 0.1 to $0.4 \mathrm{~mm}^{2-4}$.

Third, the inter-cluster correlation coefficient was 0 for all operators. This indicated that there was no difference between a single operator measuring the ONSD of a given model multiple times and multiple operators measuring the ONSD one time each. Thus, for our group of novice operators, there was no 
appreciable difference between them in conducting ultrasound for ONSD. Subsequently, one can extrapolate this to mean that amongst experienced operators there will also be no appreciable difference between their measurements of ONSD.

Despite the important results of our study, there are some limitations. First, all of the data surrounding the intra- and interoperator variability was determined in the controlled setting of a model. This was by design, in order to reduce the error of ONSD measurements found in vivo related to patient factors and time constraints. Thus, it is difficult to extrapolate the data to in vivo application. However, one could predict the variability and random error to be worse in vivo. In vivo variation could potentially be amplified by anatomical variations, due to optic nerve pathology and proptosis for example. Furthermore, in vivo variation secondary to rolling eye movements and rapid fluctuation in ICP cannot be recreated by our model. Second, the random measurement error could be secondary to our model and not the technique of ONSD ultrasonography. Though this may be true, our data supporting the very strong relation of shadow size to actual disc size suggests our model is reliable, and the error is likely associated with the operator. Finally, the use of novice operators to determine inter-operator variability may have produced data that does not extrapolate to experienced operators, however given that this technique is still considered by most to be 'experimental', finding a large cohort of 'experts' presents a challenge for this type of study.

With the creation of this novel model of ONSD for ultrasonography and the determination of intra- and interoperator variability associated with its use, we believe this is possibly a viable simulation platform for instruction of this challenging technique and that simulation should be considered prior to in vivo application of ONSD ultrasound measurement. Ideally, such standardization of technical factors will assist in the performance of a well-designed prospective clinical trial to evaluate the benefits of ONSD ultrasound to patient outcome.

\section{Conclusions}

Utilizing a novel model for ONSD ultrasonography, we have determined the intra- operator reliability of ONSD measurement to be moderate, with no appreciable difference amongst multiple operators. Improvement in measurement reliability has been demonstrated between expert and novice operators with our model, indicating the potential benefit of simulation platforms to teach the technique of ONSD ultrasound.

\section{ACKNOWLEDGEMENTS}

This research was supported by a General Operating Grant from the Health Science Centre Foundation in Winnipeg, Manitoba, Canada.

\section{REFERENCES}

1. Dubourg J, Javouhey E, Geeraerts T, Messerer M, Kassai B. Ultrasonography of optic nerve sheath diameter for detection of raised intracranial pressure: a systematic review and metaanalysis. Intensive Care Med. 2011;37:1059-68.

2. Moretti R, Pizzi B, Cassini F, Vivaldi N. Reliability of optic nerve ultrasound for the evaluation of patients with spontaneous intracranial hemorrhage. Neurocrit Care. 2009;11:406-10.

3. Bauerle J, Lochner P, Kaps M, Nedelmann M. Intra- and interobserver reliability of sonographic assessment of the optic nerve sheath diameter in healthy adults. J Neuroimaging. 2012; $22: 42-5$.

4. Ballantyne SA, O Neill G, Hamilton R, Hollman AS. Observer variation in the sonographic measurement of optic nerve sheath diameter in normal adults. Eur J Ultrasound. 2002;15:145-9.

5. McAuley D, Paterson A, Sweeney L. Optic nerve sheath ultrasound in the assessment of paediatric hydrocephalus. Childs Nerv Syst. 2009; $25: 87-90$.

6. Hansen HC, Helmke K. Validation of the optic nerve sheath response to changing cerebrospinal fluid pressure: ultrasound findings during intrathecal infusion tests. J Neurosurg. 1997;87: $34-40$

7. Rajajee V, Fletcher JJ, Rochlen LR, Jacobs TL. Comparison of accuracy of optic nerve ultrasound for the detection of intracranial hypertension in the setting of acutely fluctuating vs stable intracranial pressure: post-hoc analysis of data from a prospective, blinded single center study. Critical Care. 2012; 16:R79.

8. Zeiler FA, Unger B, Kramer AH, Kirkpatrick AW, Gillman LM. A unique model for ultrasound assessment of optic nerve sheath diameter. Can J Neurol Sci. 2013;40(2):225-9.

9. Gangemi M, Cennamo G, Maiuri F, D'Andrea F. Echographic measurement of optic nerve in patients with intracranial hypertension. Neurochirurgia (Stuttg). 1987;30(2):53-5.

10. Rajajee V, Vanaman M, Fletcher JJ, Jacobs TL. Optic nerve ultrasound for the detection of raised intracranial pressure. Neurocrit Care. 2011;15(3):506-15.

11. Strumwasser A, Kwan RO, Yeung L, et al. Sonographic optic nerve sheath diameter as an estimate of intracranial pressure in adult trauma. J Surg Res. 2011;170(2):265-71.

12. Moretti R, Pizzi B. Ultrasonography of the optic nerve in neurocritically ill patients. Acta Anaesthesiol Scand. 2011;55(6): 644-52.

13. Kimberly HH, Shah S, Marill K, Noble V. Correlation of optic nerve sheath diameter with direct measurement of intracranial pressure. Acad Emerg Med. 2008;15(2):201-4. 